\title{
Chronic morbidity, deprivation and primary medical care spending in England in 2015- 16: a cross-sectional spatial analysis
}

Evangelos Kontopantelis ${ }^{1,2^{*}}$ D, Mamas A. Mamas ${ }^{3}$, Harm van Marwijk ${ }^{1,2}$, Andrew M. Ryan ${ }^{4}$, Peter Bower ${ }^{1,2}$, Bruce Guthrie $^{5}$ and Tim Doran ${ }^{6}$

\begin{abstract}
Background: Primary care provides the foundation for most modern health-care systems, and in the interests of equity, it should be resourced according to local need. We aimed to describe spatially the burden of chronic conditions and primary medical care funding in England at a low geographical level, and to measure how much variation in funding is explained by chronic condition prevalence and other patient and regional factors.

Methods: We used multiple administrative data sets including chronic condition prevalence and management data (2014/15), funding for primary-care practices (2015-16), and geographical and area deprivation data (2015). Data were assigned to a low geographical level (average 1500 residents). We investigated the overall morbidity burden across 19 chronic conditions and its regional variation, spatial clustering and association with funding and area deprivation. A linear regression model was used to explain local variation in spending using patient demographics, morbidity, deprivation and regional characteristics.

Results: Levels of morbidity varied within and between regions, with several clusters of very high morbidity identified. At the regional level, morbidity was modestly associated with practice funding, with the North East and North West appearing underfunded. The regression model explained 39\% of the variability in practice funding, but even after adjusting for covariates, a large amount of variability in funding existed across regions. High morbidity and, especially, rural location were very strongly associated with higher practice funding, while associations were more modest for high deprivation and older age.

Conclusions: Primary care funding in England does not adequately reflect the contemporary morbidity burden. More equitable resource allocation could be achieved by making better use of routinely available information and big data resources. Similar methods could be deployed in other countries where comparable data are collected, to identify morbidity clusters and to target funding to areas of greater need.
\end{abstract}

Keywords: Primary care funding, Chronic conditions, Morbidity, Deprivation, Spatial clustering, Quality and Outcomes Framework, QOF, England, UK, Carr-Hill formula, Global sum allocation formula

\footnotetext{
*Correspondence: e.kontopantelis@manchester.ac.uk

'Division of Population Health, Health Services Research \& Primary Care; Faculty of Biology, Medicine and Health, University of Manchester, Greater Manchester, UK

${ }^{2} \mathrm{NIHR}$ School for Primary Care Research, Faculty of Biology, Medicine and Health, University of Manchester, 5th Floor Williamson Building, Greater Manchester, UK

Full list of author information is available at the end of the article
} International License (http://creativecommons.org/licenses/by/4.0/), which permits unrestricted use, distribution, and reproduction in any medium, provided you give appropriate credit to the original author(s) and the source, provide a link to the Creative Commons license, and indicate if changes were made. The Creative Commons Public Domain Dedication waiver (http://creativecommons.org/publicdomain/zero/1.0/) applies to the data made available in this article, unless otherwise stated. 


\section{Background}

Primary care plays a vital role in coordinating care and managing demand in the community, and provides the cornerstone of many modern health-care systems [1]. Systems with a strong primary- care focus are associated with lower care costs, higher patient satisfaction levels, better overall health, lower medication use and a decrease in hospitalisation and emergency department visits [2, 3]. Primary-care-focused health care can also reduce the impact of socioeconomic factors on health [3], leading to improvements in overall health and reductions in health inequalities across population subgroups [4].

One of the ongoing challenges for primary care is to tackle variability in outcomes, and to contribute towards breaking the link between wealth and health [5]. Whilst coordinated primary-care initiatives can help to reduce health inequalities, they can also unintentionally increase them: universal interventions that are effective in improving population health, such as smoking cessation programmes [6], often increase inequities because they are more effective in affluent areas [7]. Efforts to improve equity are also undermined if resources are not allocated according to need. The UK's National Health Service (NHS) is built on a strong primary-care base and provides universal comprehensive care free at the point of delivery, but striking inequities in health outcomes remain [8].

In an attempt to address this, in the early 2000s the UK government pursued a range of interventions intended to improve equity and reformed the method of funding primary-care practices. A new national General Medical Services contract for general practitioners was introduced in 2004, with two main funding components. Core funding for essential and key additional services was calculated using the Carr-Hill global sum formula $[9,10]$, based on the number of registered patients, adjusted for patient factors (including age, sex, turnover, morbidity and mortality) and the local context (staff market forces and rurality). This core funding was supplemented by a new pay-for-performance programme - the Quality and Outcomes Framework (QOF) - introduced with the aim of increasing overall funding for primary care and reducing variation in quality between providers [11]. QOF payments were dependent on practice performance against over 100 quality targets relating to practice organisation, patient experience and clinical management of chronic conditions. Payments for clinical targets were adjusted according to the relative prevalence of the relevant condition in the practice population. Further investment in primary care was targeted at deprived areas in 2007 and 2008 [12], with the aim of supporting local efforts to tackle chronic disease and increasing physician numbers in areas where physician recruitment and health-care delivery can be challenging [13].
For several reasons, these initiatives did not fully address the inequitable distribution of resources and its contribution towards the perpetuation of health inequalities [14]. First, funding under the QOF programme initially favoured larger practices in more affluent areas, which tended to perform better against the quality targets $[15,16]$. Gaps in performance rapidly narrowed [15, 17], although there was no clear evidence for improvement in patient outcomes [18], or impact on mortality over the longer term $[5,19]$. Second, payment adjustments intended to protect practice incomes had unintended consequences. For the clinical QOF targets, relative prevalence was calculated based on the square root of disease prevalence rather than prevalence itself, and practices with low disease prevalence - below the 5 th percentile - were treated as if prevalence were equal to the 5th percentile. These adjustments were intended to retain parity between practices with respect to quality payments, but had the effect of uncoupling the relationship between workload and remuneration, disadvantaging practices with high disease prevalence, which were more likely to be in deprived areas. For this reason, from 2009 onwards the prevalence adjustment was calculated based on actual prevalence. Third, the global sum formula for core funding does not directly adjust for patient deprivation and uses a measure of morbidity based on Standardised Limited Long-Standing Illness data derived from the 1998-2000 Health Survey for England [9]. There are long-standing concerns that this formula does not fully reflect the pressures and costs that deprivation imposes on practices [20]. In response to these concerns, under the 2004 contract, practices received a Minimum Practice Income Guarantee (MPIG), a correction payment to prevent their core funding, based on the new global sum formula, falling below historical levels. MPIG began to be phased out in 2014 with aim of equalising weighted funding per patient across all practices by 2021 $[20,21]$. This has left many practices in deprived areas facing financial hardship and urgently calling for a fairer system of resource allocation [21]. NHS England, NHS Employers and the British Medical Association are committed to revising the Carr-Hill formula to reflect deprivation better [10,22], but agreement on a new system of allocation has yet to be reached.

Since the introduction of the QOF in 2004, annually updated prevalence data have been available for numerous chronic conditions at the practice level, and these could provide more precise, timely and comprehensive information for determining health-care need. In this study, we aimed to describe the overall chronic condition burden, as measured by prevalence data derived from the 2014/15 QOF, and to evaluate its association with primary medical care funding in England for 201516. More specifically, we aimed to (a) spatially describe 
the overall chronic condition burden and payments to primary-care practices at a low geographical level; (b) quantify and describe the variability and spatial clustering in these two measures, across ten English regions and (c) measure how much of the variation in spending is explained by the overall chronic condition burden and how much by other relevant population factors measurable at a low geographical level, in particular area deprivation.

\section{Methods}

Our primary unit of analysis was the Lower Layer Super Output Area (LSOA) in England: 32,844 geographical administrative units with an average population of 1500 . Deprivation was measured though the 2015 Index of Multiple Deprivation (IMD) [23]. To measure the morbidity burden we created a chronic morbidity index (CMI), calculated as the sum of 19 chronic condition registers in the 2014/15 QOF, divided by the total practice population. Unfortunately, the measure cannot capture comorbidity since that information is not recorded within the QOF. Someone with two conditions would be entered independently in the two respective registers. NHS payments to general practices for 2015-16 were reported by NHS Digital, covering all centrally managed payment schemes (global sum, MPIG, balance of PMS (Personal Medical Services) expenditure, QOF and enhanced services) and also the decentralised Local Enhanced Services scheme. Although other local payments were not captured (for example, Local Authority public health funding) the reported payments were in effect the bulk of the income for general practices [24]. Both funding and the CMI were assigned to LSOAs using methodology previously described [12]. Further details of the data and the methods used to attribute them to the LSOA level are provided in Additional file 1: Appendix 1.

\section{Analyses}

The outcome of interest was average primary medical funding per patient in 2015-16. The key covariates were the IMD 2015 and the CMI of the 2014/15 QOF measured at the LSOA level. Correlations between the IMD and the CMI were calculated using Pearson's rho for the whole of England and each of the ten regions (at the LSOA level, weighted for 2014 population estimates). Funding and the CMI were visualised using spatial maps for all of England and each region. After aggregating at the regional level (weighting for 2014 LSOA populations), scatter plots were used to describe the relationship between the outcome and each of the two key covariates. Box plots were plotted to describe the distribution of funding and CMI within each region.
Spatial autocorrelation for funding per person, deprivation and morbidity (correlation in a signal among nearby locations in space) was assessed and quantified using Moran's $I$. This measure accounts for the multidimensional and multi-directional nature of spatial autocorrelation, and can identify the presence of clusters. A higher value than the one expected under a random spatial pattern would indicate that, using morbidity as an example, areas with high levels of morbidity are clustered together and, hence, a high morbidity LSOA is more likely to be bordered by (or have as close neighbours) LSOAs with similarly high morbidity levels. We calculated Moran's $I$ for each region and the whole of England, to allow for within-England comparisons.

A linear regression model, weighted for 2015 LSOA size, was used to quantify the strength of association between average primary medical care funding per person and region, demographic characteristics (age, sex and ethnicity), urbanity, the CMI and the IMD. A second model with interaction terms is discussed in Additional file 1: Appendix 1. Our choice between unadjusted (denominator $=$ attribution population) and adjusted (denominator $=$ LSOA population) per patient cost was informed by the predictive power of the regression models, with the former metric leading to better models. Hence, all analyses and graphs we present use unadjusted funding.

Analyses were executed with Stata v14.1 and R v3.3.1. Most comparisons are statistically significant due to the size of the data set and thus, we focus on effects sizes where possible. All variables we used were complete.

\section{Results}

A total of 56,924,424 patients were registered with a general practice in January 2015. Total funding for practices in $2015-16$ was $£ 7.61$ billion. Based on the number of registered patients, the median annual primary medical care funding per patient, minus prescription and dispensing costs, was $£ 133.7$ at the LSOA level [interquartile range (IQR), $£ 123.5$ to $£ 148.0$ ] (Table 1 and Fig. 1). Total median regional spending per person varied from $£ 125.4$ in South Central to $£ 145.7$ in Yorkshire and the Humber. Based on population estimates rather than registers, funding per person was higher, at $£ 140.4$ (IQR, £128.4 to £156.9). The median CMI for England was 0.51 conditions at the LSOA level (IQR, 0.45 to 0.57 ), with the highest levels observed in the North East (median, 0.59; IQR, 0.54 to 0.62 ) and the lowest in London (median, 0.38; IQR, 0.34; 75th centile, 0.42). Correlations between the IMD and the CMI, weighted for LSOA population sizes in 2014 (rather than 2015, since the CMI is based on 2014/15 QOF data), were very weak for each region and close to zero for England. The largest (absolute) rho was observed for the 


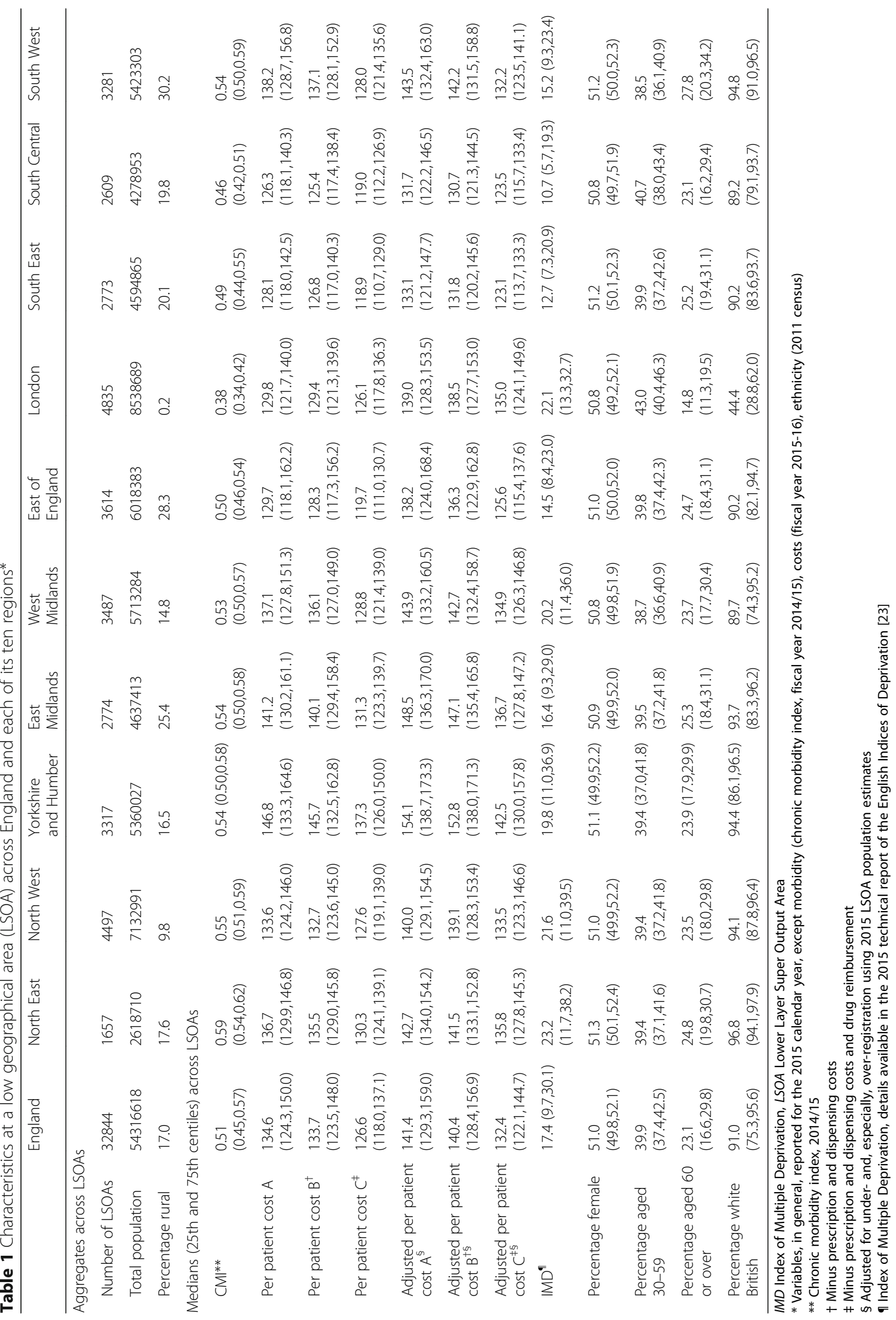



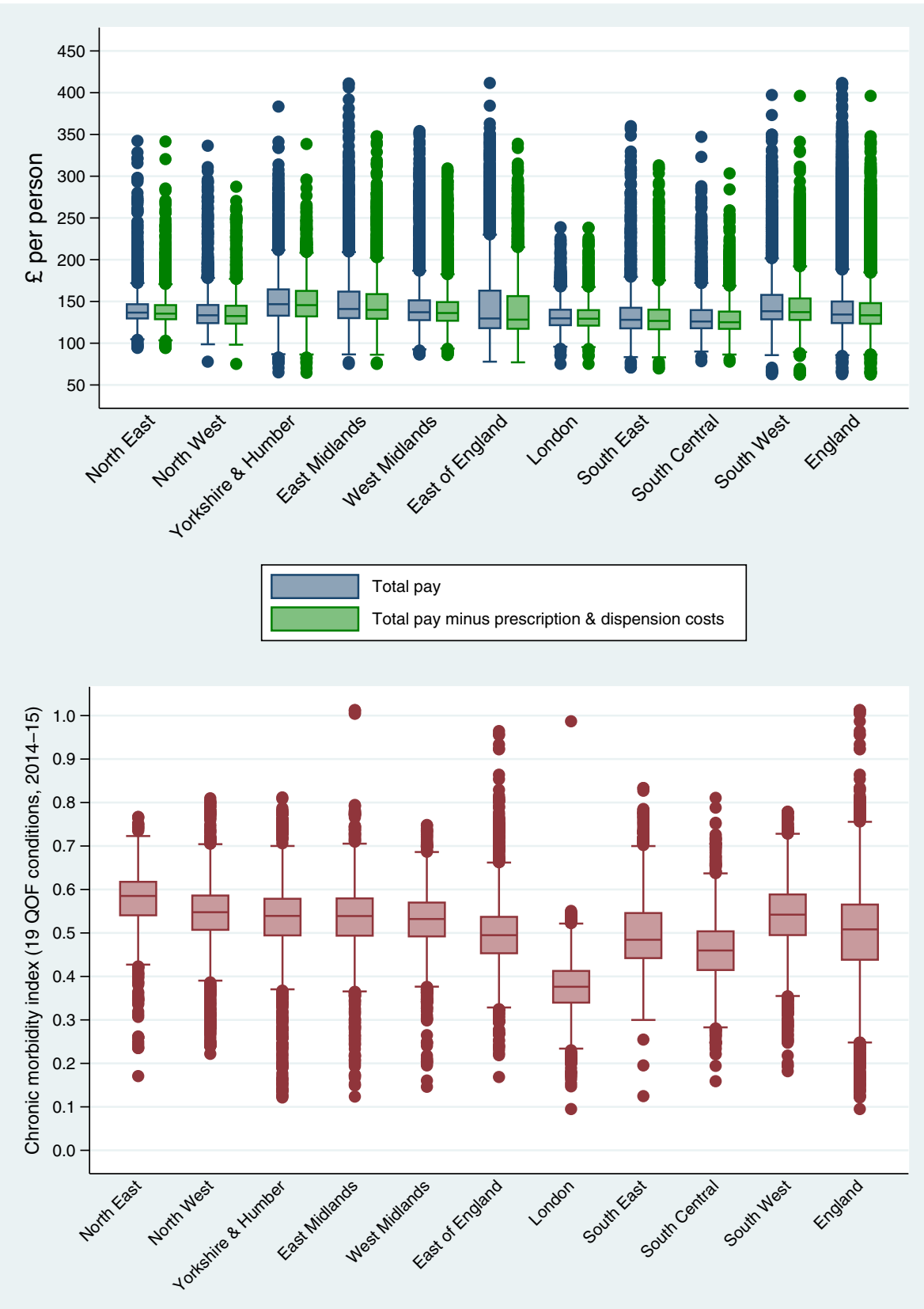

Fig. 1 Box plots of average primary medical care spending for 2015-16 (top) and the chronic morbidity index for 2014/15 (bottom), across English regions, weighted for LSOA population sizes. LSOA Lower Layer Super Output Area, QOF Quality and Outcomes Framework

West Midlands (-0.194), and the rho for the whole of England was 0.004 .

The spatial variability of the CMI and average funding per person are presented in Figs. 2 and 3, respectively (regional spatial maps are also provided in Additional file 2: Appendix 2). We observed great variation in the CMI both across and within regions. Very high levels of morbidity were concentrated in large areas in the North East, the East Midlands and the East of England. London and parts of its neighbouring regions (South Central, South East and East of England) that are close to the capital consistently had the lowest CMI levels. For average funding per person, we also observed great regional variability, which did not necessarily match the CMI patterns. For example, there were very low levels of funding for many parts of the North West and funding for the region as a whole was relatively low compared to other regions - whereas the overall 


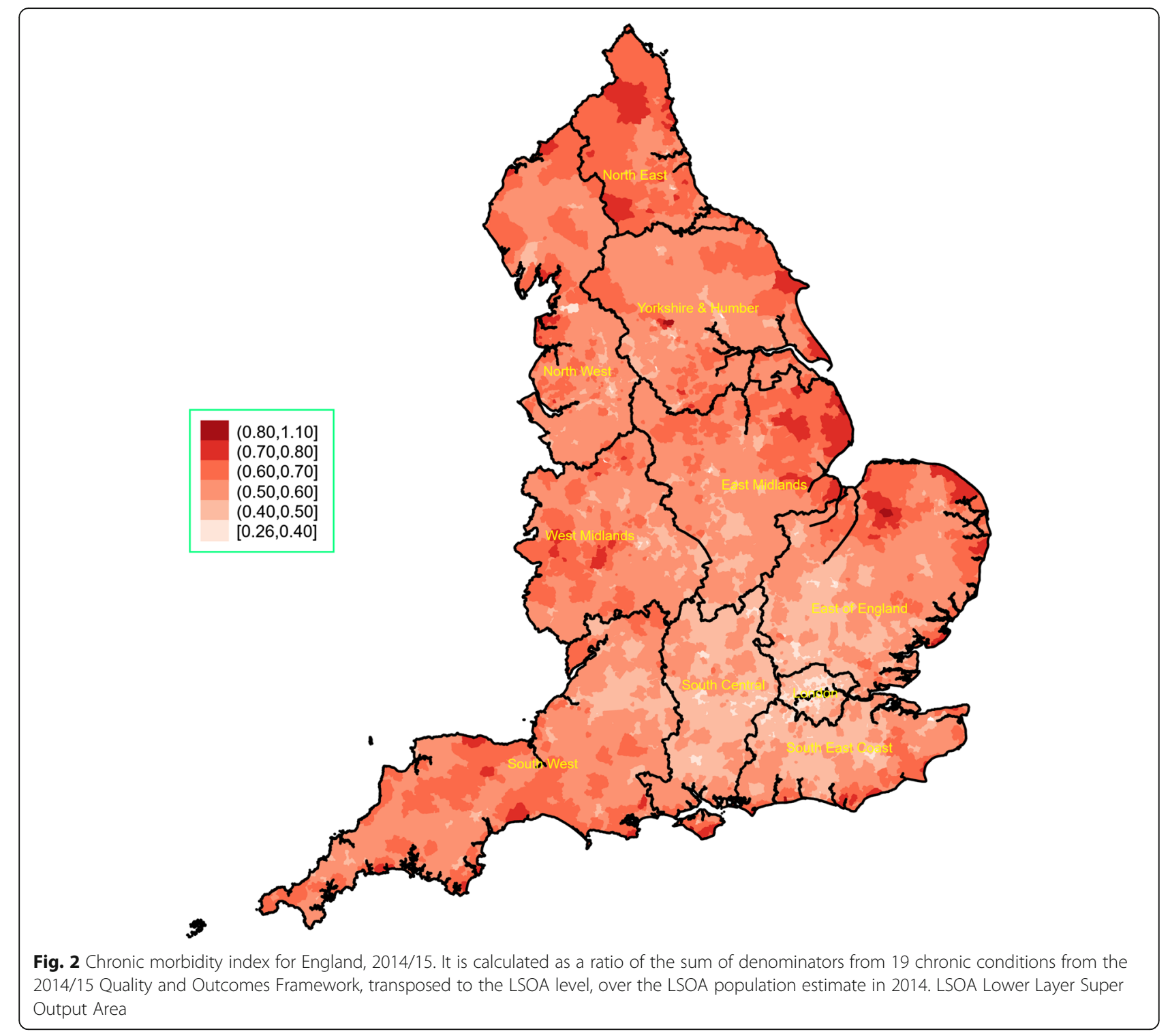

CMI level in the region was the second highest nationally. We also calculated the ratio of CMI over average funding per person $(\times 1000)$, which is plotted in Fig. 4 and highlights clusters of variability in every region and a pattern of higher ratios in the North West and the North East.

The associations between funding per person and CMI, and between funding per person and IMD, are illustrated at the regional level in Fig. 5 (plots at different geographic levels are provided in Additional file 3: Appendix 3). Increasing funding per person is associated with increasing levels of the CMI, with outliers at high and low levels of CMI (London, with the lowest level of CMI, has relatively high funding per person, and the North East and the North West regions, with the highest CMI levels, have relatively low funding). The association between funding per person and IMD is unclear, and IMD levels do not appear to be related to primary medical care funding at the regional level.

The regional variability of spatial autocorrelation for the CMI, the IMD and funding is presented in Fig. 6 . For the CMI, we observed high regional variability in spatial autocorrelation, ranging between 0.147 for London and 0.471 for the South West. This indicates that London has the least clustering of areas with similar levels of CMI, and the South West has the greatest clustering. For the IMD, spatial autocorrelation levels were lower and had less variation, ranging from 0.109 in London to 0.194 in the South East. Levels of spatial autocorrelation were also modest for funding, but there was greater variability between regions, ranging from 0.074 in the West Midlands to 0.242 in the East of England. 


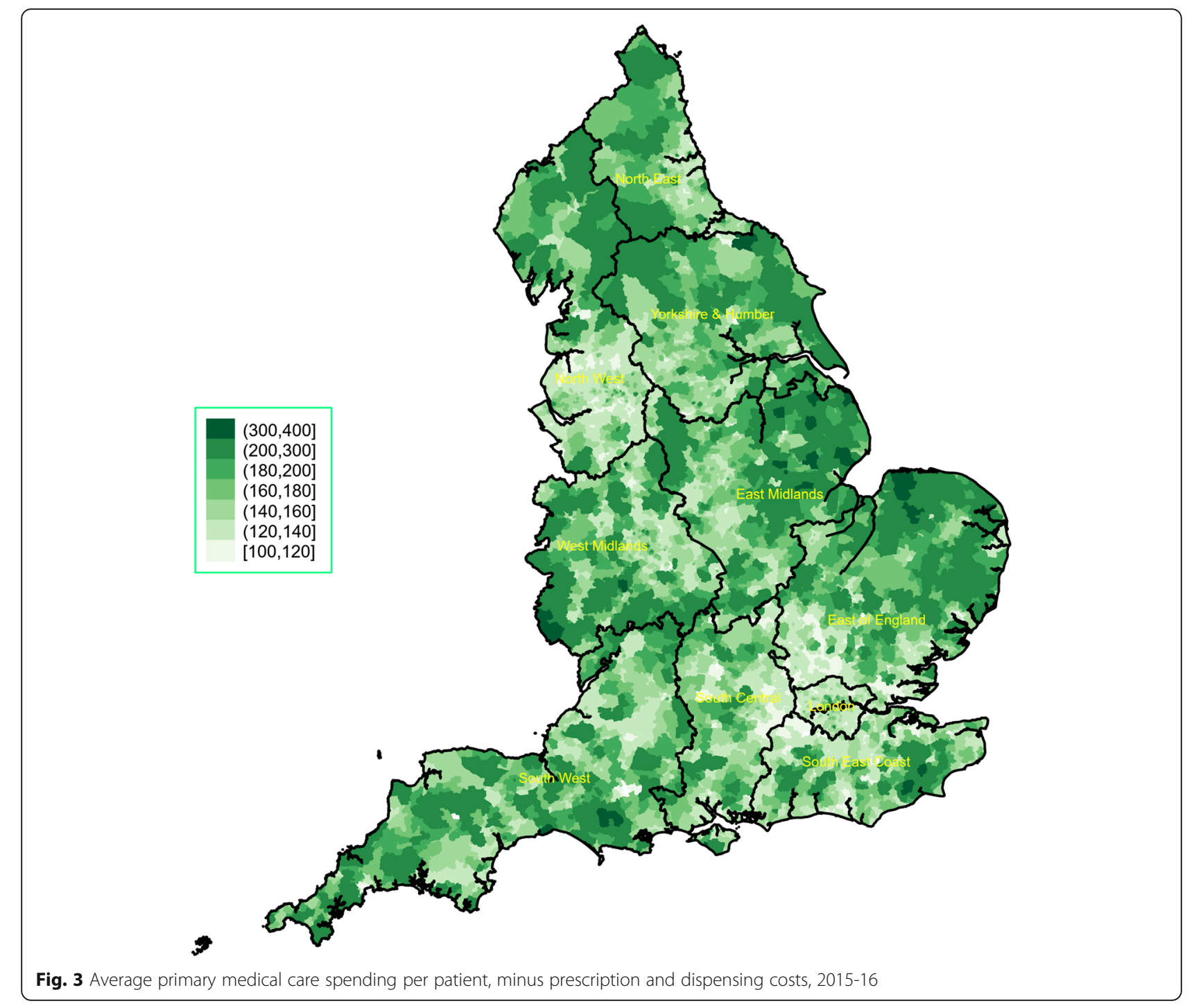

After adjusting for demographics, urbanity, the CMI and the IMD, there was large variability in funding between regions (Table 2). Compared to Yorkshire and the Humber, the region with the highest average funding per person, adjusted funding in the South East was $£ 14.50$ [95\% confidence interval (CI), 13.38 to 15.62 ] lower per person. The CMI was a strong predictor of funding, with a 0.1 -unit increase, i.e. from 0.513 (50th centile) to 0.613 (89th centile), associated with a $£ 8.12$ (95\% CI, 7.71 to $8.54)$ increase in funding per person. The IMD was also a strong predictor with a 10 -unit increase in IMD, i.e. from 17.4 (50th centile) to 27.4 (71st centile), associated with a $£ 2.26$ (95\% CI, 2.06 to 2.45 ) increase in funding per person. The strongest independent predictor was urbanity, with rural areas associated with $£ 35.71$ (95\% CI, 35.02 to 36.41 ) higher funding per person. Other large associations were observed for LSOAs with a large population over 60 and more females. An increase of
$10 \%$ in patients over 60 within an LSOA (i.e. from $23.1 \%$ or the 50 th centile to $33.1 \%$ or the 84 th centile) was associated with a $£ 2.54$ (95\% CI, 2.12 to 2.95 ) increase per person. The association for females is weaker than it appears because sex distribution is generally well balanced. An increase of $1 \%$ in females, i.e. an increase from $51 \%$ (50th centile) to $52 \%$ (73rd centile), was associated with a $£ 0.49$ (95\% CI, $0.39,0.59)$ decrease per person.

\section{Discussion}

Primary-care funding in England is only modestly associated with morbidity burden, as measured by the CMI at the regional level, with the North East and North West having the highest levels of morbidity but only average levels of funding. We observed great variability in the CMI both within and between regions, with clusters of very high morbidity burden in the North East, East Midlands and East England. 


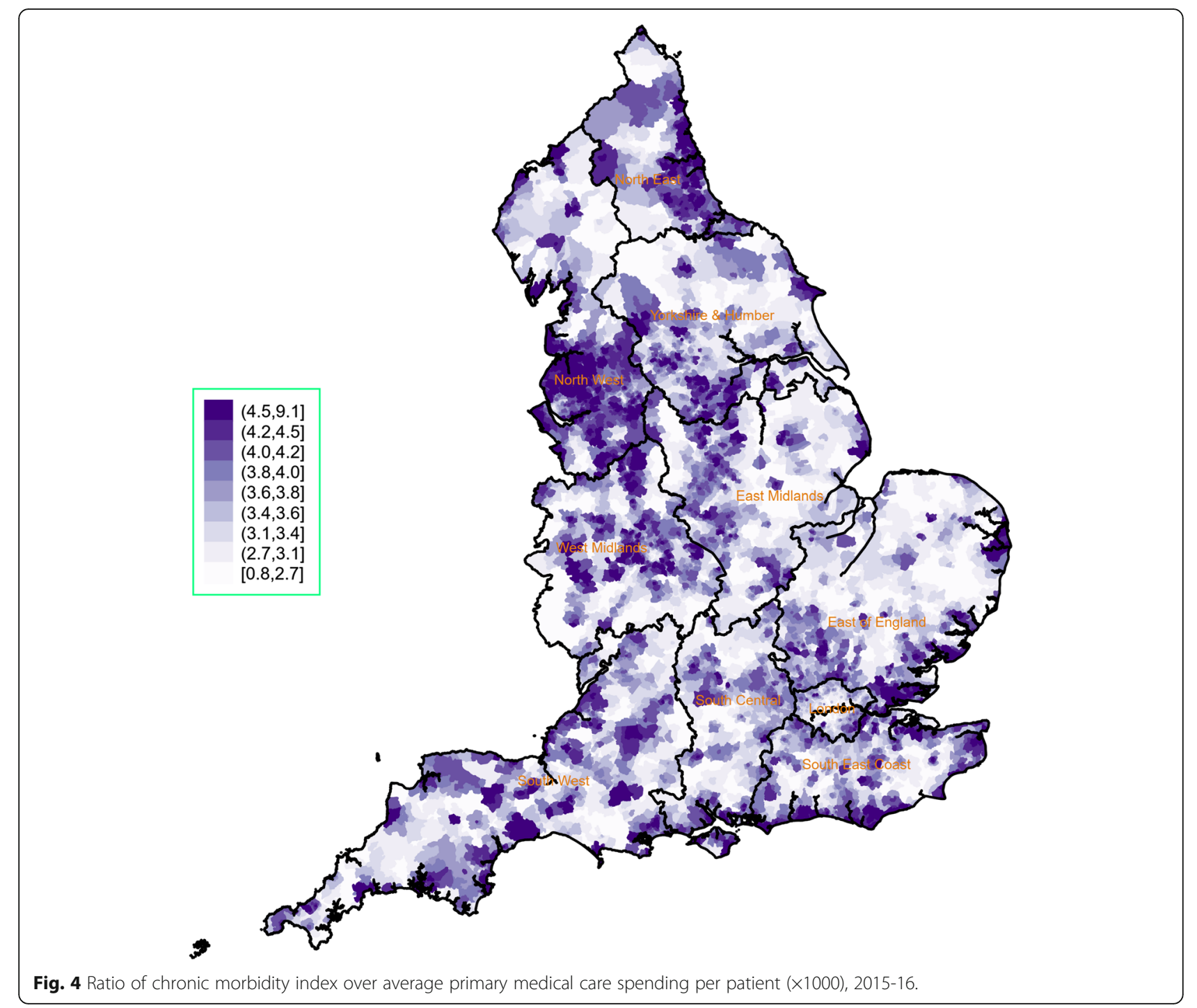

Spatial clustering of the CMI was high, especially for the South West, and was not associated with area deprivation in the patient locality, while funding was more uniformly distributed across space. Although morbidity, deprivation and age were strong predictors of funding, the strongest predictor was rurality, with less funding per person in urban centres. The model explained a reasonable level of variability in funding (39\%), but even after adjusting for covariates, there was large variability between regions, which was also observed in relation to the strength of the associations between morbidity and funding, and between deprivation and funding.

\section{Strengths and limitations of the study}

The strength of the study lies in the quality of the databases and their sizes. We investigated the whole of England and over 55 million people being served by a universal health system. Through their electronic health records, we calculated a measure of morbidity, the CMI, and linked it to funding per person. The CMI is designed to capture overall health needs for 19 common chronic conditions rather than multi-morbidity, but we would expect it to be a strong proxy for multi-morbidity.

The study has the potential for ecological fallacy, with practice-level information assigned to geographies. Although, we have assessed that risk and found it to be low in terms of deprivation (see Additional file 1: Appendix 1), the assignment could be improved by using age-sex stratification weights at the LSOA level. A second limitation is that ethnicity and urbanity information was available for 2011 and we necessarily assumed there has been little change over time. Although this will not be the case for all regions and LSOAs, the 2001 and 2011 versions of these variables were very strongly correlated, indicating little change over time. A third limitation is that 


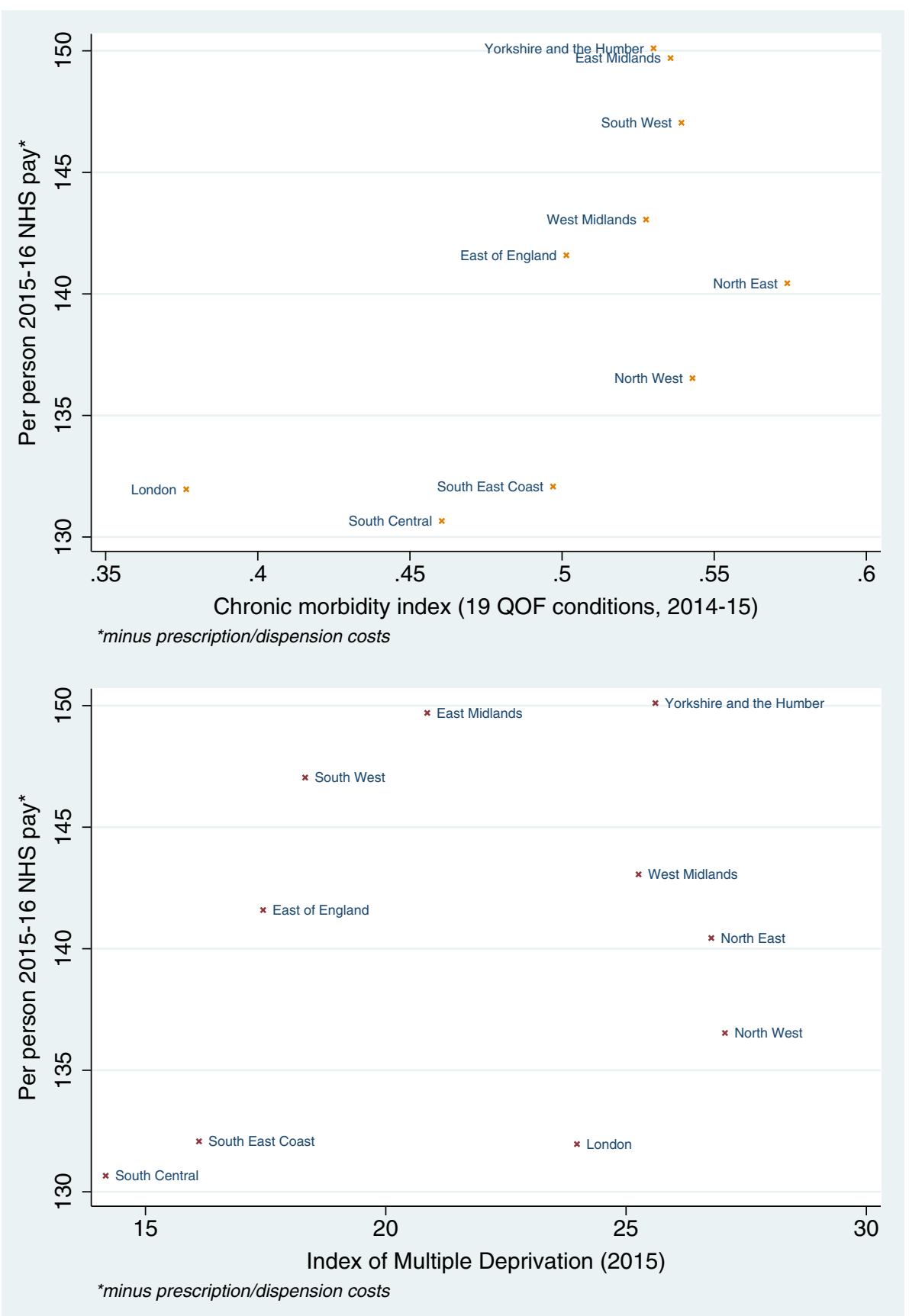

Fig. 5 Scatter plot of average primary medical care spending for 2015-16 by chronic morbidity index (top) and the 2015 Index of Multiple Deprivation (bottom), across English regions, weighted for LSOA population sizes. LSOA Lower Layer Super Output Area, QOF Quality and Outcomes Framework

we could not directly assess multi-morbidity in any form, due to the way the data are collected in England. It has been shown in Scotland that multimorbidity is higher in deprived areas and is associated with higher demand but not additional funding [25]. Fourth, our analysis is dependent on accurate diagnosis and recording of conditions within primary care. There is a danger that any future system of resource allocation based on practice registers would incentivise practices to inflate these registers. Finally, to weight the regression models for LSOA sizes, we necessarily used standard regression models rather than spatialautoregressive models, which may have affected our estimates. However, the estimated spatial autocorrelation for the whole of England is low and should not affect the precision of our model estimates. 


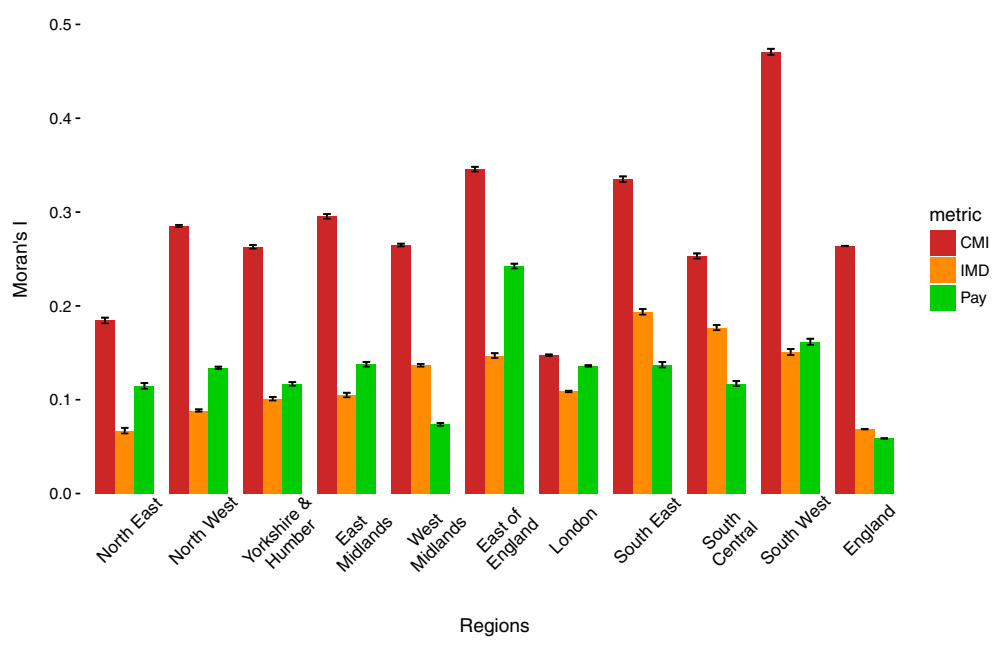

Fig. 6 Spatial clustering (Moran's / with 95\% confidence intervals) for 2015-16 average primary medical care pay (minus prescription and dispensing costs, per person), the 2014/15 chronic morbidity index (CMI) and the 2015 Index of Multiple Deprivation (IMD), within each region and for the whole of England. CMI chronic morbidity index, IMD Index of Multiple Deprivation

Table 2 Results from model A, linear regression at the LSOA level*†

\begin{tabular}{|c|c|c|c|c|}
\hline & Coefficient & \multicolumn{2}{|c|}{ 95\% confidence interval } & $p$ value \\
\hline \multicolumn{5}{|l|}{ Region } \\
\hline Yorkshire and Humber & reference & & & \\
\hline North East & -13.62 & -14.93 & -12.30 & $<0.001$ \\
\hline North West & -12.47 & -13.46 & -11.47 & $<0.001$ \\
\hline East Midlands & -3.42 & -4.53 & -2.32 & $<0.001$ \\
\hline West Midlands & -6.59 & -7.64 & -5.54 & $<0.001$ \\
\hline East England & -8.83 & -9.88 & -7.78 & $<0.001$ \\
\hline London & -0.43 & -1.64 & 0.78 & 0.484 \\
\hline South East & -14.50 & -15.62 & -13.38 & $<0.001$ \\
\hline South Central & -12.38 & -13.54 & -11.21 & $<0.001$ \\
\hline South West & -7.71 & -8.78 & -6.65 & $<0.001$ \\
\hline \multicolumn{5}{|l|}{ Demographics } \\
\hline Percentage aged 30-59, 2015 & 0.040 & -0.013 & 0.093 & 0.140 \\
\hline Percentage aged 60 or over, 2015 & 0.254 & 0.212 & 0.295 & $<0.001$ \\
\hline Percentage female, 2015 & -0.489 & -0.592 & -0.387 & $<0.001$ \\
\hline Percentage white British, 2011 & -0.067 & -0.084 & -0.049 & $<0.001$ \\
\hline \multicolumn{5}{|l|}{ Urbanity } \\
\hline Rural LSOA & 35.71 & 35.02 & 36.41 & $<0.001$ \\
\hline \multicolumn{5}{|l|}{ Morbidity and deprivation } \\
\hline Chronic morbidity index & 81.22 & 77.08 & 85.36 & $<0.001$ \\
\hline IMD 2015 & 0.226 & 0.206 & 0.245 & $<0.001$ \\
\hline constant & 118.34 & 112.80 & 123.89 & $<0.001$ \\
\hline
\end{tabular}

* 32,844 LSOAs (observations) with analytic weighting

† Adjusted $R$-squared $=38.85 \%$ 
The low-level geographical approach includes advantages as well as risks. It allows us to control analyses for population characteristics that are linked to the small area geography, especially area deprivation. It also enables us to plot detailed spatial maps to visualise the parameters of interest and to identify geographical clusters of high and low need. These analyses can inform future service reorganisations and, by identifying areas where the observed morbidity load is lower than expected, uncover possible under-diagnosis and unmet need [26].

\section{Findings}

We failed to identify a strong association between the overall morbidity burden, as measured by the recorded prevalence of QOF conditions, and area deprivation. Although multi-morbidity levels are positively associated with deprivation [27], and the onset of multi-morbidity occurs much earlier in people living in the most deprived areas [28], our findings are not contradictory. First, mean age is lower in deprived inner-city areas, which offsets the unadjusted association between deprivation and morbidity. Second, although associations between deprivation and prevalence rates have been observed for conditions such as diabetes even when not adjusted for age [29], these balance out across the whole spectrum of conditions (at the practice level, Pearson's rho $=0.05)$. However, this may also be an indication of under-diagnosis in more deprived areas and better case finding and recording in more affluent areas.

Regional variability in the CMI highlights the varying levels of prevalence for QOF conditions across England. The highest CMI levels were observed in the North East (median 0.59 conditions per person) and the lowest in London (median 0.38 conditions, reflecting its younger population). Our findings are broadly in agreement with previous reports for individual conditions at a higher geography [30], considering we report an aggregate measure. However, our low-geography mapping approach facilitates within-region investigations and the identification of geographical clusters of high disease prevalence. Large clusters of very high prevalence were observed for the North East, East Midlands and East England, and additional resources or reorganisation of services may be needed to serve these populations better [for example, extended opening hours or redistribution of general practitioners (GPs)]. Levels of spatial clustering were particularly high for the South West (there were no large high-prevalence clusters but there was a high level of spatial variation across the whole region), which could also inform the organisation of care. Importantly, although regional CMI levels were broadly associated with average primary medical care funding per person, the North East, North West and London appear to be outliers, with fewer than expected resources allocated to the North East and the North West, and more to London. In particular for Greater Manchester, and its very recently devolved health and social care spending, a £2bn a year funding gap is expected by 2020 if demand trends are not curbed and existing barriers to efficiency and effectiveness are not removed http:// www.bmj.com/content/352/bmj.i1495.long. This imbalance between health need and resource allocation may be a contributing factor to the North-South divide in young adult mortality rates [31].

At the low-geography level, patient age, sex, ethnicity, morbidity (CMI), deprivation (IMD) and rurality explained a higher level of variation in funding than a previous model at the practice level for England [32]. More importantly, unlike in previous work at the practice level, we found the expected positive associations between funding and patient morbidity, deprivation and age. This may be an indication of the strength of our approach at the low geographical level, where area deprivation can be modelled in greater detail. However, regional variations in funding existed even after adjusting for all these parameters, with the South East, North East, North West and South Central having the lowest levels of adjusted funding. As expected, morbidity, deprivation and age were strong predictors of funding. Rurality was also a very strong independent predictor, with rural areas receiving an adjusted average of $£ 35.70$ more per patient. Prescribing and dispensing costs have been suggested as explaining this disparity, but these costs were excluded from our analyses. The higher cost seems to be at least partially driven by the smaller list sizes in practices located in rural areas (on average 831 fewer patients), while staffing levels are similar in both rural (average full-time equivalent of 4.2 GPs and 2.3 nurses) and urban settings (average full-time equivalent of 4.1 GPs and 2.1 nurses).

\section{Conclusions}

To meet societal goals of providing equitable health care, funding for primary-care systems must be distributed according to need, fully accounting for the impact of deprivation. We have described the morbidity burden in England at a low geographical level using routinely collected administrative data. Not only have we identified unexplained regional variation in common morbidities, we have also found evidence that the current allocation of resources to primary care does not account for all important health needs. More optimal resource utilisation in primary care in the UK is required [25], and better use of the wealth of information resources already available could help to achieve this aim through the design of fairer resource allocation formulae. It is, therefore, vital that the disease registers introduced as part of the QOF are retained and updated when the scheme is phased out. 
Similar information is also routinely collected in other developed countries, and the methods we have described can be highly relevant, both in identifying disease clusters or under-diagnosis, and in matching resources to need. Findings from such analyses are used in countries that use public-sector allocation mechanisms, whether central (e.g. Ireland and France) or devolved (e.g. Denmark, Sweden and Spain), to directly inform resource allocation. In countries with insurance-based mechanisms (e.g. France, Germany and the Netherlands), analyses can be used to identify mismatches between allocation and need, which may require government intervention through risk equalisation schemes [33] or alternative mechanisms. This will require difficult decisions about the fundamental aims of resource allocation and within a limited health budget - a commitment to tackling oversupply as well as undersupply [34].

\section{Additional files}

Additional file 1: Appendix 1. Details of methods and additional

analyses. (DOCX $47 \mathrm{~kb}$ )

Additional file 2: Appendix 2. Spatial maps by English region.

(DOCX $3327 \mathrm{~kb}$ )

Additional file 3: Appendix 3. Scatter plots for deprivation vs. funding and morbidity vs. funding. (DOCX $325 \mathrm{~kb}$ )

\section{Acknowledgments}

We would like to thank the Office of National Statistics and NHS Digital (formerly the Health and Social Care Information Centre) for the wealth of information they have collected and systematically organised, which made this study possible. We would also like to thank the reviewers whose comments and suggestions greatly improved this manuscript.

\section{Funding}

A Health eResearch Centre grant from the Medical Research Council (MR/ K006665/1) supported the time and facilities of EK. No specific funding was received for this study and no funding bodies had any role in study design, data collection and analysis, decision to publish, or preparation of the manuscript.

\section{Availability of data and materials}

The data used in this study are freely available and the authors are happy to share the organised and cleaned final data set.

\section{Authors' contributions}

EK designed the study, extracted the data from all sources and performed the analyses. EK and TD wrote the manuscript. MM, PB, BG, AMR and HVM critically revised the manuscript. EK is the guarantor of this work and, as such, had full access to all the data in the study and takes responsibility for the integrity of the data and the accuracy of the data analysis. All authors read and approved the final manuscript.

\section{Ethics approval and consent to participate}

Not applicable.

\section{Consent for publication}

Not applicable.

\section{Competing interests}

The authors declare that they have no competing interests.

\section{Publisher's Note}

Springer Nature remains neutral with regard to jurisdictional claims in published maps and institutional affiliations.

\section{Author details}

${ }^{1}$ Division of Population Health, Health Services Research \& Primary Care; Faculty of Biology, Medicine and Health, University of Manchester, Greater Manchester, UK. ${ }^{2}$ NIHR School for Primary Care Research, Faculty of Biology, Medicine and Health, University of Manchester, 5th Floor Williamson Building, Greater Manchester, UK. ${ }^{3}$ Science and Technology in Medicine, Keele University, Staffordshire, UK. ${ }^{4}$ School of Public Health, University of Michigan, Ann Arbor, MI, USA. ${ }^{5}$ Population Health Sciences Division, School of Medicine, University of Dundee, Dundee, UK. ${ }^{6}$ Department of Health Sciences, University of York, Yorkshire, UK.

Received: 25 August 2017 Accepted: 15 December 2017

Published online: 14 February 2018

\section{References}

1. Starfield B. Is primary care essential? Lancet. 1994;344(8930):1129-33.

2. Starfield B. Primary care: concept, evaluation, and policy. New York: Oxford University Press; 1992.

3. Shi L. The impact of primary care: a focused review. Scientifica. 2012;2012: 432892.

4. Starfield B, Shi LY, Macinko J. Contribution of primary care to health systems and health. Milbank Q. 2005;83(3):457-502.

5. Kontopantelis E, Springate DA, Ashworth M, Webb RT, Buchan IE, Doran $\mathrm{T}$. Investigating the relationship between quality of primary care and premature mortality in England: a spatial whole-population study. $\mathrm{Br}$ Med J. 2015;350:h904

6. Mons U, Muezzinler A, Gellert C, Schottker B, Abnet CC, Bobak M, et al. Impact of smoking and smoking cessation on cardiovascular events and mortality among older adults: meta-analysis of individual participant data from prospective cohort studies of the CHANCES consortium. Br Med J. 2015;350:h1551.

7. Statistics on smoking: England 2015. https://digital.nhs.uk/catalogue/ PUB17526. Accessed 1 June 2017.

8. Dixon A, Le Grand J, Henderson J, Murray R, Poteliakhoff E. Is the British National Health Service equitable? The evidence on socioeconomic differences in utilization. J Health Serv Res Policy. 2007;12(2):104-9.

9. BMA. focus on the global sum allocation formula (Carr-Hill Formula). England: BMA; 2015.

10. Elliot R, Sutton M, Gravelle H, Hole A, Ma A, Morris S, et al. Adjusting the General Medical Services Allocation Formula to reflect recruitment and retention difficulties. 2006.

11. Roland M. Linking physicians' pay to the quality of care - a major experiment in the United Kingdom. N Engl J Med. 2004;351(14):1448-54.

12. Asaria M, Ali S, Doran T, Ferguson B, Fleetcroft R, Goddard M, et al. How a universal health system reduces inequalities: lessons from England. J Epidemiol Commun H. 2016;70(7):637-43.

13. Young $R$, Leese $B$. Recruitment and retention of general practitioners in the UK: what are the problems and solutions? Br J Gen Pract. 1999;49(447):829-33.

14. Goddard M, Smith P. Equity of access to health care services: theory and evidence from the UK. Soc Sci Med. 2001;53(9):1149-62.

15. Doran T, Fullwood C, Kontopantelis E, Reeves D. Effect of financial incentives on inequalities in the delivery of primary clinical care in England: analysis of clinical activity indicators for the Quality and Outcomes Framework. Lancet. 2008;372(9640):728-36.

16. Doran T, Campbell S, Fullwood C, Kontopantelis E, Roland M. Performance of small general practices under the UK's Quality and Outcomes Framework. Br J Gen Pract. 2010;60(578):e335-44.

17. Ashworth M, Medina J, Morgan M. Effect of social deprivation on blood pressure monitoring and control in England: a survey of data from the Quality and Outcomes Framework. Br Med J. 2008;337:a2030.

18. Gillam SJ, Siriwardena AN, Steel N. Pay-for-performance in the United Kingdom: impact of the Quality and Outcomes Framework: a systematic review. Ann Fam Med. 2012;10(5):461-8.

19. Ryan AM, Krinsky S, Kontopantelis E, Doran T. Long-term evidence for the effect of pay-for-performance in primary care on mortality in the UK: a population study. Lancet. 2016;388(10041):268-74. 
20. Boomla K, Hull S, Robson J. Is general practice in trouble? GP funding formula masks major inequalities for practices in deprived areas. BMJ. 2014;349:g7648. https://doi.org/10.1136/bmj.g7648.

21. lacobucci G. MPIG set to be phased out within seven years in sweeping overhaul of GP funding. In: PULSE. London: 2012

22. England N. General practice forward view. 2016. London: NHS England; 2016. p. 60.

23. Communities and Local Government: The English Indices of Deprivation 2015: Technical Report. In. London: Department for Communities and Local Government; 2015

24. NHS Payments to General Practice, England, 2015-16. http://content.digital. nhs.uk/catalogue/PUB21318. Accessed 1 June 2017.

25. McLean G, Guthrie B, Mercer SW, Watt GCM. General practice funding underpins the persistence of the inverse care law: cross-sectional study in Scotland. Br J Gen Pract. 2015;65(641):E799-805.

26. Soljak M, Samarasundera E, Indulkar T, Walford H, Majeed A. Variations in cardiovascular disease under-diagnosis in England: national cross-sectional spatial analysis. BMC Cardiovasc Disor. 2011;11:12.

27. Salisbury C, Johnson L, Purdy S, Valderas JM, Montgomery AA. Epidemiology and impact of multimorbidity in primary care: a retrospective cohort study. Br J Gen Pract. 2011;61(582):e12-21. https://doi.org/10.3399/ bjgp11X548929.

28. Barnett K, Mercer SW, Norbury M, Watt G, Wyke S, Guthrie B. Epidemiology of multimorbidity and implications for health care, research, and medical education: a cross-sectional study. Lancet. 2012:380(9836):37-43

29. Car J, Millett C, Khunti K, Mainous A, Majeed A. Diabetes prevalence, process of care and outcomes in relation to practice size, caseload and deprivation: national cross-sectional study in primary care. Diabetic Med. 2007;24:78.

30. Public Health England. The NHS atlas of variation in healthcare. 2016. p. 284.

31. Buchan IE, Kontopantelis E, Sperrin M, Chandola T, Doran T. North-South disparities in English mortality1965-2015: longitudinal population study. J Epidemiol Community Health. 2017;71(9):928-36.

32. Levene LS, Baker R, Wilson A, Walker N, Boomla K, Bankart MJG. Population health needs as predictors of variations in NHS practice payments: a crosssectional study of English general practices in 2013-2014 and 2014-2015. Br J Gen Pract. 2017;67(654):E10-19.

33. van de Ven WPMM, Beck K, van de Voordec C, Wasem J, Zmora I. Risk adjustment and risk selection in Europe: 6 years later. Health Policy. 2007; 83(2-3):162-79.

34. Buck D, Dixon A. Improving the allocation of health resources in England. London: Kings Fund; 2013.

\section{Submit your next manuscript to BioMed Central and we will help you at every step:}

- We accept pre-submission inquiries

- Our selector tool helps you to find the most relevant journal

- We provide round the clock customer support

- Convenient online submission

- Thorough peer review

- Inclusion in PubMed and all major indexing services

- Maximum visibility for your research

Submit your manuscript at www.biomedcentral.com/submit 